\title{
Hyperthermic Intravesical Chemotherapy for BCG Unresponsive Non-Muscle Invasive Bladder Cancer Patients
}

\author{
Joep J. de Jong ${ }^{\mathrm{a}}$, Kees Hendricksen ${ }^{\mathrm{b}}$, Marloes Rosier ${ }^{\mathrm{a}}$, Hugh Mostafid ${ }^{\mathrm{c}}$ and Joost L. Boormans ${ }^{\mathrm{a}, *}$ \\ ${ }^{a}$ Department of Urology, Erasmus MC Cancer Institute, Erasmus Medical Center, Rotterdam, The Netherlands \\ ${ }^{\mathrm{b}}$ Department of Surgical Oncology, Division of Urology, Netherlands Cancer Institute - Antoni van Leeuwenhoek \\ Hospital, Amsterdam, The Netherlands \\ ${ }^{\mathrm{c}}$ Department of Urology, Royal Surrey County Hospital, Guildford, UK
}

Received 2 July 2018

Accepted 7 August 2018

\begin{abstract}
.
Background: Adjuvant intravesical instillations with bacillus Calmette-Guérin (BCG) is the recommended treatment option for patients with intermediate- and high-risk non-muscle invasive bladder cancer (NMIBC). Despite adequate BCG treatment, a large proportion of patients experience a recurrence. Although radical cystectomy is the gold standard for BCG unresponsive NMIBC, some patients are unfit or unwilling to consider this option.

Objective: To assess the effectiveness of Hyperthermic IntraVEsical Chemotherapy $\left(\right.$ HIVEC $\left.^{\circledR}\right)$ in BCG unresponsive NMIBC patients.

Methods: A post-hoc analysis was conducted of prospectively included intermediate- and high-risk NMIBC patients who were planned to receive HIVEC ${ }^{\circledR}$ treatment between October 2014 and November 2017. For the present analysis, only patients who met the BCG unresponsive definition were included. Patients were followed by cystoscopy and cytology every 3 months and a CT-urography scan yearly. The primary outcome was the disease-free survival (DFS). The Common Terminology Criteria for Adverse Events (CTCAE) was used to assess side-effects.

Results: The study population consisted of 55 BCG unresponsive NMIBC patients of whom 52 underwent $\geq 5$ HIVEC ${ }^{\circledR}$ treatments. The median age and follow-up were 73 years and 14.0 months (IQR 7.6-24.6). The median DFS was 17.7 months (SE 6.72) and progression occurred in four patients. The 1-year cumulative incidence rate of disease recurrence/progression was 53\%. Two patients experienced severe side-effects (CTCAE $\geq 3$ ).

Conclusions: HIVEC $^{\circledR}$ seems a valid treatment option for BCG unresponsive NMIBC patients. We report a median DFS of 17.7 months (SE 6.72), potentially avoiding or postponing the need for radical surgery in a proportion of these patients.
\end{abstract}

Keywords: BCG unresponsive, BCG, bladder cancer, chemo-hyperthermia

\section{INTRODUCTION}

The global incidence of bladder cancer is 430,000 patients per year [1]. Roughly $75 \%$ of patients present with non-muscle invasive bladder cancer (NMIBC)

\footnotetext{
${ }^{*}$ Correspondence to: J.L. Boormans, MD, PhD, Erasmus MC Cancer Institute, Department of Urology, Room Na1512, P.O. Box 2040, 3000 CA Rotterdam, The Netherlands. Tel.: +31 107040704; Fax:+31 107044762; E-mail: j.boormans@erasmusmc.nl.
}

[2]. Adjuvant intravesical instillations with bacillus Calmette-Guérin (BCG) is the recommended treatment option for patients with intermediate- and high-risk NMIBC [3]. Treatment with BCG typically includes a 6 -week induction course followed by 3 weekly instillations at 3, 6, 12, 18, 24, 30 and 36 months. This BCG maintenance regimen has shown to reduce the risk of recurrence in intermediateand high-risk NMIBC patients $[4,5]$. Nonetheless, 
approximately $50 \%$ of patients will develop a recurrence within 5 years after BCG treatment and even a proportion of patients experience progression to muscle invasive bladder cancer (MIBC), which negatively impacts survival [6-8]. Some patients experience recurrent disease despite adequate BCG treatment, which is defined as having had $\geq 5$ of 6 weekly induction instillations followed by $\geq 2$ additional instillations of maintenance therapy or a second reinduction course of 6 BCG instillations. In addition, patients can experience disease recurrence because they did not receive adequate BCG treatment due to BCG intolerance or shortage [9]. A number of concepts have been published of how disease recurrence during or after BCG treatment should be defined [10-14]. Consequently, comparing salvage therapies in patients failing BCG has been hindered by the lack of standard definitions and studies that combined different classes of BCG-failure. Therefore, the International Bladder Cancer Group (IBCG) recently defined BCG unresponsive NMIBC to truly define patients who fail BCG. BCG unresponsive disease includes patients who experience high grade tumor recurrence within 6-9 months from the last BCG exposure despite adequate BCG treatment [9]. The prognosis of BCG unresponsive NMIBC patients is poor and radical cystectomy is currently the recommended treatment option in these patients [15]. However, many patients have comorbid conditions, are elderly or are simply not willing to undergo radical surgery. The optimal therapy in such cases is unknown, as established and effective salvage intravesical therapies are not yet available [15]. The European Association of Urology and International Consultation on Urologic Diseases (EAU-ICUD) does not endorse salvage intravesical gemcitabine, valrubicin, or IFN $\alpha$, in patients who fail BCG, but chemo-hyperthermia is recommended as a promising treatment modality [16]. The objective of the present study was to assess the effectiveness and safety of hyperthermic intravesical chemotherapy $\left(\right.$ HIVEC $^{\circledR}$ ) in BCG unresponsive NMIBC patients, who were unfit or unwilling to undergo immediate radical cystectomy.

\section{MATERIALS AND METHODS}

Study design

A post-hoc analysis was conducted of prospectively included intermediate- and high-risk NMIBC (urothelial carcinoma) patients who were planned to receive HIVEC $^{\circledR}$ treatment between October 2014 and November 2017 at three institutions: Erasmus Medical Center, Rotterdam, the Netherlands, the Netherlands Cancer Institute, Amsterdam, the Netherlands and the Royal Surrey County Hospital, Guildford, United Kingdom. Protocol has been approved by a local Institutional Review Board, under number MEC-2015-077. Patients were eligible for inclusion in the present study if: i) the criteria for BCG unresponsive disease were met: i.e. adequate BCG treatment (at least 5/6 induction and 2/3 maintenance instillations) and high grade tumor within 6 to 9 months of the last BCG exposure [9], ii) had histologically confirmed high-grade NMIBC (WHO classification system 1973: Grade 2 or 3, WHO 2014: High-grade), including papillary NMIBC alone (Ta or T1), carcinoma in situ (CIS), or a combination of CIS and papillary disease.

\section{Treatment schedule}

The HIVEC ${ }^{\circledR}$ treatment schedule at the Erasmus Medical Center consisted of 10 intravesical instillations (four weekly induction instillations, followed by six monthly maintenance instillations). The treatment schedule at the Netherlands Cancer Institute and the Royal Surrey County Hospital consisted of 12 intravesical instillations (six weekly induction instillations, followed by six monthly maintenance instillations). Intravesical instillations with Mitomycin-C (MMC) were administered at a concentration of $80 \mathrm{mg}$ diluted in $50 \mathrm{~mL}$ of distilled water. The solution was extravesically heated up to $41-43^{\circ} \mathrm{C}$ and recirculated during $60 \mathrm{~min}$ at $200 \mathrm{~mL}$ per min at stable pressure. All instillations were conducted with the Combat BRS system V2.0, which was used according to manufacturer's instructions (Combat Medical, Wheathampstead, UK). Only patients who completed at least 5 HIVEC $^{\circledR}$ instillations were included in the effectiveness analysis. Safety was analyzed in all patients.

\section{Follow-up, primary outcome and statistical analysis}

All patients were followed by cystoscopy and cytology every three months and biopsies were performed if cystoscopy showed a recurrence or cytology showed a suspicion of the presence of urothelial carcinoma. A CT-scan of the urinary tract was performed once a year. The primary outcome was the disease-free survival (DFS), defined as the 
time from start of first HIVEC ${ }^{\circledR}$ treatment until disease recurrence and/or progression. Recurrence was defined as histologically confirmed diagnosis of high-grade urothelial carcinoma, while progression was defined as histologically or radiologically confirmed diagnosis of MIBC or metastatic disease. For patients presenting with (concomitant) CIS, the secondary outcome was the complete response (CR) at 3 months, defined as the absence of CIS, high-grade disease or progression by cystoscopy and cytology or biopsy. The Kaplan-Meier method was used to generate estimates of DFS. Patients were censored at the date of disease recurrence/progression or date of last cystoscopy. Safety end points included: type, incidence, severity of adverse events (AEs) and severe ( $\geq$ grade 3) AEs (SAEs), as assessed by the Common Terminology Criteria for Adverse Events v5.0 (CTCAE). Adverse events were reported by the clinicians of each participating center. Risk of tumor recurrence was estimated by the cumulative incidence of recurrence/progression at 3,6,9 and 12 months. Statistical analysis was performed using IBM SPSS statistics for windows, version 24 .

\section{RESULTS}

A total of 55 BCG unresponsive NMIBC patients who were planned to receive HIVEC $^{\circledR}$ treatment were included. Table 1 lists the baseline characteristics of the patient cohort. Three patients had not completed at least 5 HIVEC $^{\circledR}$ instillations, so 52 patients were included in the effectiveness analyses. The median follow-up was 14.0 months (IQR 7.6 - 24.6) and did not significantly differ between patients who remained disease free $(\mathrm{N}=26)$, and patients who experienced recurrence or progression $(\mathrm{N}=26)$ during follow-up (12.8 vs 19.0 months, respectively) $(P=0.41)$. The overall median DFS was 17.7 months (SE 6.72) (Fig. 1a). The median DFS in patients with papillary disease only $(\mathrm{N}=22)$ was 28.8 months (SE 11.9), while the median DFS in patients having (concomitant) CIS $(\mathrm{N}=30)$ was 17.7 months (SE 6.1) (Fig. $1 b, P=0.55$ ). The median DFS in 'very high risk' BCG unresponsive patients ( $\mathrm{T} 1$ or $\mathrm{T} 1+\mathrm{CIS}, \mathrm{N}=12$ ) was 12.1 months (SE 4.6) (Fig. 1c). At 3 months, 21 out of $30(70 \%)$ patients having (concomitant) CIS had a $\mathrm{CR}$. The 1-year cumulative incidence rate of disease recurrence/progression was 53\%. Table 2 lists the cumulative incidence rates at the 3,6,9 and 12 months of follow-up.
Table 1

Patient and tumor characteristics of 55 BCG unresponsive NMIBC patients

\begin{tabular}{lc}
\hline Baseline characteristics $(\mathrm{N}=55)$ & \\
\hline Age (median \& range) & $73(34-87 \mathrm{yrs})$ \\
Hospital & $21(38 \%)$ \\
Erasmus Medical Center & $17(31 \%)$ \\
Netherlands Cancer Institute & $17(31 \%)$ \\
Royal Surrey County Hospital & \\
Gender & $48(87 \%)$ \\
Male & $7(13 \%)$ \\
Female & \\
T-stage & $15(27 \%)$ \\
Ta & $9(16 \%)$ \\
T1 & $1(2 \%)$ \\
Ta + CIS & $3(6 \%)$ \\
T1 + CIS & $27(49 \%)$ \\
CIS only & \\
Tumor Grade & $4(7 \%)$ \\
G2 (HG) & $24(44 \%)$ \\
G3 & $27(59 \%)$ \\
CIS only & \\
EAU Risk group & $55(100 \%)$ \\
High & \\
BCG & $55(100 \%)$ \\
Unresponsive & \\
HIVEC instillations & $52(95 \%)$ \\
$\geq 5$ & $3(5 \%)$ \\
$<5$ &
\end{tabular}

\section{Therapy for disease recurrence or progression}

Overall, 26 patients $(50 \%)$ remained disease free, 22 patients (42\%) experienced recurrent disease and four patients (8\%) experienced progression to MIBC or metastatic disease during followup. Of the 22 patients experiencing recurrent disease, $11(50 \%)$ underwent radical cystectomy (pathology showed: pTisN0 in 7, pT1N0 + CIS in 2, pT3aN0 in one, and for one patient we could not retrieve pT-stage because he underwent cystectomy in another hospital. The last patient is currently planned for radical cystectomy. Four patients received rechallenge treatment with $\mathrm{BCG}$, four remained under endoscopic surveillance, one patient underwent curative external beam radiotherapy and one patient received intravesical gemcitabine. Of the four patients who had progressive disease, one underwent palliative chemotherapy, one underwent palliative chemotherapy with radiotherapy, one is currently undergoing neo-adjuvant systemic chemotherapy and one received best supportive care due to liver metastases (Table 3). Bladder cancer related death during follow-up occurred in two patients. 

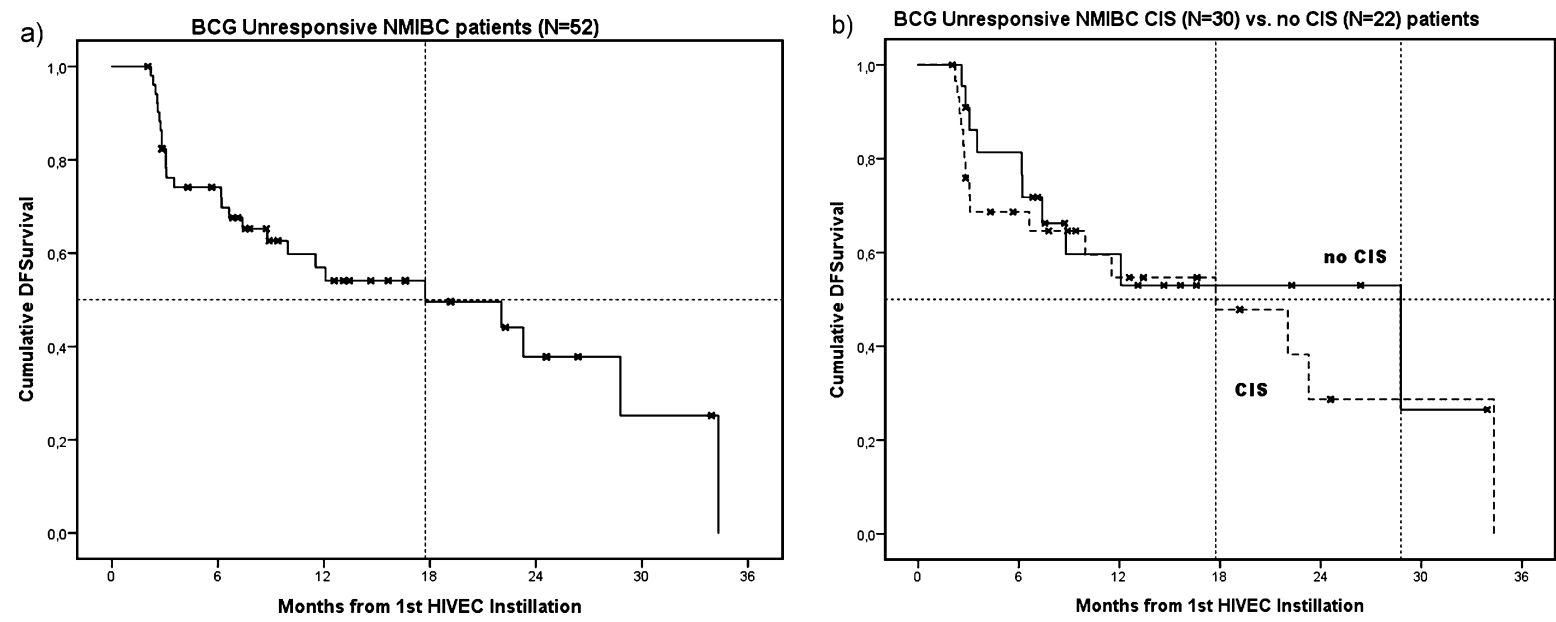

Log-Rank test: $P=0.545$

C) Very high risk (T1/T1 + CIS) BCG unresponsive NMIBC patients ( $N=12)$

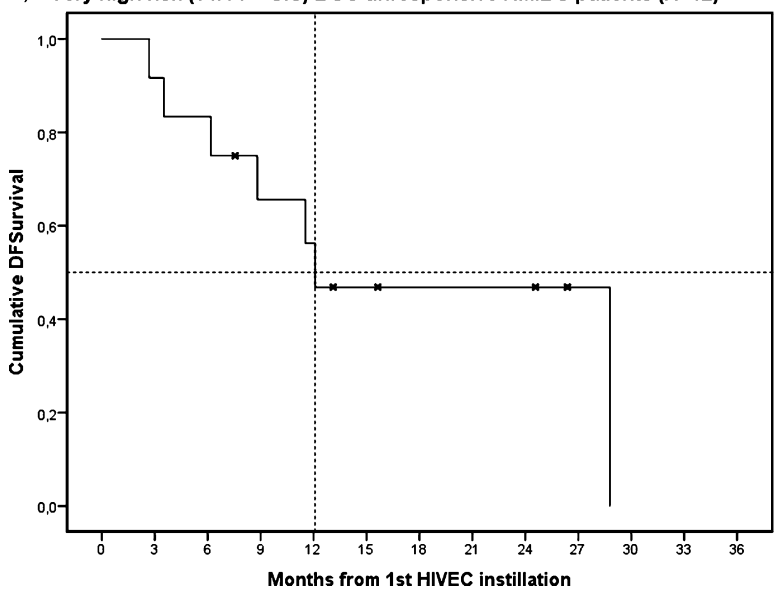

Fig. 1. Kaplan Meier curves showing DFS after HIVEC ${ }^{\circledR}$ treatment in BCG unresponsive NMIBC patients (a), BCG unresponsive NMIBC CIS versus no CIS patients (b) and ‘very high risk' BCG unresponsive NMIBC patients (c).

Table 2

Cumulative incidence rates of patients who underwent $\geq 5$ HIVEC $^{\circledR}$ instillations $(\mathrm{N}=52)$ at $3,6,9$ and 12 months of follow-up

\begin{tabular}{lcccc}
\hline $\begin{array}{l}\text { Follow-up } \\
\text { moment }\end{array}$ & $\begin{array}{c}\text { No of recurrent } \\
\text { patients }\end{array}$ & $\begin{array}{c}\text { Patients still } \\
\text { under FU }\end{array}$ & $\begin{array}{c}\text { Cum. Patients } \\
\text { censored }\end{array}$ & $\begin{array}{c}\text { Cum. Incidence } \\
\text { rate }\end{array}$ \\
\hline 3 months & 13 (6 persistent CIS) & 52 & 0 & $25 \%(13 / 52)$ \\
6 months & 4 & 48 & 4 & $35 \%(17 / 48)$ \\
9 months & 2 & 43 & 9 & $44 \%(19 / 43)$ \\
12 months & 2 & 40 & 12 & $53 \%(21 / 40)$ \\
\hline
\end{tabular}

\section{Adverse events}

Of the 55 patients, three $(5 \%)$ did not complete at least 5 HIVEC $^{\circledR}$ instillations, because of an $\mathrm{AE}$ - facial swelling, urticaria, and urinary tract pain, respectively). For 38 patients (69\%) at least one $\mathrm{AE}$ during the instillation course was reported, whereas 17 patients $(31 \%)$ had no side effects. Table 4 lists all experienced AEs. All treatment-related AEs were CTCAE graded and no patients had grade IV or V AEs. Two SAEs ( $\geq$ grade 3 ) were reported (severe urinary tract pain and a urinary tract infection for which IV antibiotics were indicated) and occurred among the patients who did complete a minimum of 5 HIVEC $^{\circledR}$ instillations. In the patient with severe urinary tract pain, HIVEC $^{\circledR}$ treatment was stopped 
Table 3

Treatment modality for patients who experienced recurrence or progression under $\mathrm{HIVEC}^{\circledR}$ treatment $(\mathrm{N}=26)$

\begin{tabular}{lc}
\hline Treatment modality & No of patients (\%) \\
\hline Recurrent disease $(\mathrm{N}=22)$ & $11(50 \%)$ \\
Radical Cystectomy & $1(4 \%)$ \\
Planned for Radical Cystectomy & $4(19 \%)$ \\
BCG rechallenge & $4(19 \%)$ \\
Endoscopic surveillance & $1(4 \%)$ \\
Curative external beam radiotherapy & $1(4 \%)$ \\
Intravesical gemcitabine & \\
Progressive disease $(\mathrm{N}=4)$ & $1(25 \%)$ \\
Neo-adjuvant chemotherapy & $1(25 \%)$ \\
Palliative chemotherapy & $1(25 \%)$ \\
Palliative chemotherapy + radiotherapy & $1(25 \%)$ \\
Best supportive care & \\
\hline
\end{tabular}

Table 4

Reported adverse events during HIVEC ${ }^{\circledR}$ instillations

\begin{tabular}{|c|c|}
\hline Adverse events $(\mathrm{N}=55)$ & No of patients (\%) \\
\hline No side effects reported & $17(31 \%)$ \\
\hline \multicolumn{2}{|l|}{ Bladder spasms } \\
\hline Grade 1 & $4(7 \%)$ \\
\hline \multicolumn{2}{|l|}{ Urinary frequency/urgency } \\
\hline Grade 1 & $6(11 \%)$ \\
\hline Grade 2 & $13(24 \%)$ \\
\hline \multicolumn{2}{|l|}{ Urinary tract infection } \\
\hline Grade 2 & $4(7 \%)$ \\
\hline Grade 3 & $1(2 \%)$ \\
\hline \multicolumn{2}{|l|}{ Urinary tract pain } \\
\hline Grade 1 & $2(4 \%)$ \\
\hline Grade 2 & $5(9 \%)$ \\
\hline Grade 3 & $1(2 \%)$ \\
\hline \multicolumn{2}{|l|}{ Rash acneiform } \\
\hline Grade 1 & $4(7 \%)$ \\
\hline \multicolumn{2}{|l|}{ Urticaria } \\
\hline Grade 1 & $1(2 \%)$ \\
\hline \multicolumn{2}{|l|}{ Flu-like symptoms } \\
\hline Grade 1 & $3(5 \%)$ \\
\hline \multicolumn{2}{|l|}{ Abdominal pain } \\
\hline Grade 1 & $1(2 \%)$ \\
\hline \multicolumn{2}{|l|}{ Edema face } \\
\hline Grade 1 & $1(2 \%)$ \\
\hline \multicolumn{2}{|l|}{ Dry skin } \\
\hline Grade 1 & $1(2 \%)$ \\
\hline
\end{tabular}

after the fifth instillation. It settled in two weeks with conservative treatment. The patient with severe urinary tract infection was successfully treated with IV antibiotics. Hematuria was reported among all patients with a urinary tract infection. One patient experienced a transient ischemic attack during the course of HIVEC $^{\circledR}$ treatment, but not on the day of instillation, so this event was considered as not directly related to treatment.

\section{DISCUSSION}

BCG unresponsive NMIBC is a clinical challenge in daily urologic practice because of the high risk of recurrence and progression. The gold standard for BCG unresponsive NMIBC is radical cystectomy, which is a surgical procedure with high morbidity rates. Many patients therefore seek bladder sparing cancer management. So, the management of BCG unresponsive NMIBC has been identified as an unmet clinical need by the FDA [15]. We report a median DFS of 17.7 months and a 1-year cumulative incidence rate of $53 \%$ in 52 BCG unresponsive NMIBC patients, who were treated with HIVEC $^{\circledR}$. Furthermore, data from previous studies suggest that immediate cystectomy after BCG failure is not strictly mandatory, but rather that modest and efficient radical cystectomy after an attempt at salvage intravesical therapy can be an effective strategy [15]. Many salvage therapy options have already been investigated as alternatives to radical surgery in the BCG failure setting [17, 18], but the various definitions of BCG failure precluded comparison of studies. Implementing a uniform definition for BCG unresponsiveness is necessary to be able to make a comparison between single armed trials, evaluating the efficacy of new agents after BCG immunotherapy. Therefore, the IBCG defined BCG unresponsive disease with the aim to encourage uniformity in clinical trial design. Furthermore, given the high risk of disease recurrence, a placebo-controlled arm is not ethical in BCG unresponsive disease, so experimental single armed trials with new agents are now being conducted [19]. Kamat et al. stated that agents showing DFS rates of $30 \%$ at 12 months in such trials are considered to have a clinically meaningful magnitude of effect [9]. In the present study, we report a $53 \%$ cumulative incidence, meaning $47 \%$ of the patients being disease-free at 12 months of follow-up (Table 2). As compared to other agents investigated in BCG unresponsive trials, HIVEC $^{\circledR}$ has a similar performance. For example, Shore et al. reported $35 \%$ of the patients remaining free of high-grade recurrence at 1 year after intravesical $\mathrm{rAd}-\mathrm{IFN} \alpha / \mathrm{Syn} 3$ treatment, and $\mathrm{Li}$ et al. reported a DFS of $34.8 \%$ at 1 year with Mycobacterium phlei Cell wall-Nucleic Acid complex (MCNA) [20, 21]. Valrubicin is currently the only Food and Drug Administration (FDA) approved intravesical agent, although only approved for BCG-refractory CIS, showing a modest DFS rate of approximately $10 \%$ at 12 months [22].

Hyperthermia is thought to have several beneficial effects on the treatment of bladder cancer with MMC, such as increasing the penetration of MMC into the urothelium and increasing its cytotoxicity [23, 
24]. In their meta-analysis, Lammers et al. showed 59\% less recurrences after chemo-hyperthermia compared with MMC alone in NMIBC patients [25]. The most studied application of chemo-hyperthermia is the Synergo HT $^{\circledR}$ system, in which local hyperthermia is administered via microwave irradiation of the urothelium. During HIVEC ${ }^{\circledR}$, MMC is extravesically heated by the Combat BRS system and recirculated, maintaining a high bladder temperature. No randomized comparisons between both systems have been performed yet. Several studies with proportions of BCG failure patients included using the Synergo $\mathrm{HT}^{\circledR}$ system, have been performed [26-29]. The overall reported 1-year DFS rates were higher than reported in the present study, but how many of these patients failing BCG therapy, actually met the definition of BCG unresponsive NMIBC is unclear. In a study conducted by Witjes et al. 45 out of $49(92 \%)$ patients having (concomitant) CIS, had a CR at 3 months after treatment with the Synergo $\mathrm{HT}^{\circledR}$ system, but this was not a BCG unresponsive CIS population [30]. Sousa et al. studied the efficacy of HIVEC ${ }^{\circledR}$ in a heterogeneous NMIBC population and showed a 4-year cumulative incidence of recurrence (CIR) of $20.8 \%$ in patients who received HIVEC ${ }^{\circledR}$ as a neoadjuvant treatment (before transurethral resection of a bladder tumor (TURB)) versus a 2-year CIR of $12.5 \%$ in patients who received HIVEC ${ }^{\circledR}$ as adjuvant therapy (post-TURB) [31]. However, how many patients from their cohort met the BCG unresponsive definition was also not defined.

Regarding the HIVEC ${ }^{\circledR}$ safety profile, most AEs were mild or moderate $(\mathrm{CTCAE} \leq 2)$ in severity and only 3 patients did not complete at least 5 HIVEC $^{\circledR}$ instillations, because of an AE. No patients had grade IV or V AEs and in two patients SAEs ( $\geq$ grade $3)$ were reported. The observed treatment related AE rate $(69 \%)$ is comparable to instillations with MCNA (65.9\%) and lower than intravesical rAdIFN $\alpha /$ Syn 3 treatment $(85 \%)$ in BCG unresponsive NMIBC patients [20, 32].

Our study has several limitations. First, patients were retrospectively classified to the criteria of BCG unresponsive disease. So, in cases of inaccuracies on the number and regimen of BCG treatments prior to referral, patients had to be excluded, leading to a reduced sample size. However, the data on the 52 included patients was all prospectively recorded, so documentation during follow-up was accurately conducted. Second, the three participating hospitals did not apply a similar regimen for HIVEC ${ }^{\circledR}$ treatment. However, both regimens applied the first 5 instillations weekly, which made us use this as a criterion when considering a patient as 'treated' in the effectiveness analysis. Of note, the median DFS did not significantly differ between the two treatment regimen $(P=0.83)$. A prospective observational study on HIVEC ${ }^{\circledR}$ treatment in BCG unresponsive patients would overcome these issues.

\section{CONCLUSION}

In a cohort of 52 BCG unresponsive NMIBC patients, who received $\geq 5$ HIVEC $^{\circledR}$ instillations, including 30 patients with (concomitant) CIS, 50\% of the patients remained disease free after a median follow-up of 14.0 months. This corresponds to a median DFS of 17.7 months. HIVEC ${ }^{\circledR}$ treatment was well tolerated and only three patients had to stop treatment because of side-effects. Therefore, we conclude that HIVEC $^{\circledR}$ treatment might be a feasible option in BCG unresponsive NMIBC patients, potentially avoiding or postponing the need for radical surgery in a proportion of these patients.

\section{ACKNOWLEDGMENTS}

JJJ received the Van Walree Grant of the Royal Netherlands Academy of Arts and Sciences to present the abstract at the 2018 annual meeting of the American Urological Association in San Francisco, CA, May 18-21, 2018.

\section{CONFLICT OF INTEREST}

JJJ received travel grants from Combat medical to attend scientific meetings, KH, MR and HM have no conflicts of interest to report, JLB received travel grants from Combat medical to attend scientific meetings and has received honoraria by MSD, Roche, BMS and Janssen Pharmaceuticals for consultancy work. Combat medical had no participation in the design of the study, the collection of the data and data analysis, and writing process of this manuscript.

\section{REFERENCES}

[1] Antoni S, Ferlay J, Soerjomataram I, Znaor A, Jemal A, Bray F. Bladder Cancer Incidence and Mortality: A Global Overview and Recent Trends. Eur Urol. 2017;71:96-108.

[2] Kamat AM, Hahn NM, Efstathiou JA, Lerner SP, Malmstrom PU, Choi W, et al. Bladder cancer. Lancet. 2016;388:2796-810.

[3] Babjuk M, Bohle A, Burger M, Capoun O, Cohen D, Comperat EM, et al. EAU Guidelines on Non-Muscle-invasive 
Urothelial Carcinoma of the Bladder: Update 2016. Eur Urol. 2017;71:447-61.

[4] Lamm DL. Preventing progression and improving survival with BCG maintenance. Eur Urol. 2000;37(Suppl 1):9-15.

[5] Oddens J, Brausi M, Sylvester R, Bono A, van de Beek $\mathrm{C}$, van Andel G, et al. Final results of an EORTC-GU cancers group randomized study of maintenance bacillus Calmette-Guerin in intermediate- and high-risk Ta, T1 papillary carcinoma of the urinary bladder: One-third dose versus full dose and 1 year versus 3 years of maintenance. Eur Urol. 2013;63:462-72.

[6] Sfakianos JP, Kim PH, Hakimi AA, Herr HW. The effect of restaging transurethral resection on recurrence and progression rates in patients with nonmuscle invasive bladder cancer treated with intravesical bacillus Calmette-Guerin. J Urol. 2014;191:341-5.

[7] Lamm D, Persad R, Brausi M, Buckley R, Witjes JA, Palou $\mathrm{J}$, et al. Defining progression in nonmuscle invasive bladder cancer: It is time for a new, standard definition. J Urol. 2014;191:20-7.

[8] Ehdaie B, Sylvester R, Herr HW. Maintenance bacillus Calmette-Guerin treatment of non-muscle-invasive bladder cancer: A critical evaluation of the evidence. Eur Urol. 2013;64:579-85.

[9] Kamat AM, Sylvester RJ, Bohle A, Palou J, Lamm DL, Brausi M, et al. Definitions, End Points, and Clinical Trial Designs for Non-Muscle-Invasive Bladder Cancer: Recommendations From the International Bladder Cancer Group. J Clin Oncol. 2016;34:1935-44.

[10] Jarow JP, Lerner SP, Kluetz PG, Liu K, Sridhara R, Bajorin $\mathrm{D}$, et al. Clinical trial design for the development of new therapies for nonmuscle-invasive bladder cancer: Report of a Food and Drug Administration and American Urological Association public workshop. Urology. 2014;83: 262-4.

[11] Brausi M, Witjes JA, Lamm D, Persad R, Palou J, Colombel $\mathrm{M}$, et al. A review of current guidelines and best practice recommendations for the management of nonmuscle invasive bladder cancer by the International Bladder Cancer Group. J Urol. 2011;186:2158-67.

[12] Martin FM, Kamat AM. Definition and management of patients with bladder cancer who fail BCG therapy. Expert Rev Anticancer Ther. 2009;9:815-20.

[13] O'Donnell MA, Boehle A. Treatment options for BCG failures. World J Urol. 2006;24:481-7.

[14] Lerner SP, Dinney C, Kamat A, Bivalacqua TJ, Nielsen M, O'Donnell M, et al. Clarification of Bladder Cancer Disease States Following Treatment of Patients with Intravesical BCG. Bladder Cancer. 2015;1:29-30.

[15] Kamat AM, Colombel M, Sundi D, Lamm D, Boehle A, Brausi $\mathrm{M}$, et al. BCG-unresponsive non-muscle-invasive bladder cancer: Recommendations from the IBCG. Nat Rev Urol. 2017;14:244-55.

[16] Burger M, Oosterlinck W, Konety B, Chang S, Gudjonsson $\mathrm{S}$, Pruthi R, et al. ICUD-EAU International Consultation on Bladder Cancer 2012: Non-muscle-invasive urothelial carcinoma of the bladder. Eur Urol. 2013;63:36-44.

[17] Lammers RJ, Witjes JA. Developments in intravesical therapy for non-muscle-invasive bladder cancer. Expert Rev Anticancer Ther. 2010;10:1903-16.

[18] Li R, Spiess PE, Kamat AM. Treatment Options for Patients with Recurrent Tumors After BCG Therapy: Are We Ignoring the Obvious? Eur Urol. 2018.

[19] Mukherjee N, Svatek RS, Mansour AM. Role of immunotherapy in bacillus Calmette-Guerin-unresponsive non-muscle invasive bladder cancer. Urol Oncol. 2018;36: 103-8.

[20] Shore ND, Boorjian SA, Canter DJ, Ogan K, Karsh LI, Downs TM, et al. Intravesical rAd-IFNalpha/Syn3 for Patients With High-Grade, Bacillus Calmette-GuerinRefractory or Relapsed Non-Muscle-Invasive Bladder Cancer: A Phase II Randomized Study. J Clin Oncol. 2017;35: 3410-6.

[21] Li R, Amrhein J, Cohen Z, Champagne M, Kamat AM. Efficacy of Mycobacterium Phlei Cell Wall-Nucleic Acid Complex (MCNA) in BCG-Unresponsive Patients. Bladder Cancer. 2017;3:65-71.

[22] Dinney CP, Greenberg RE, Steinberg GD. Intravesical valrubicin in patients with bladder carcinoma in situ and contraindication to or failure after bacillus Calmette-Guerin . Urol Oncol. 2013;31:1635-42.

[23] Paroni R, Salonia A, Lev A, Da Pozzo LF, Cighetti G, Montorsi F, et al. Effect of local hyperthermia of the bladder on mitomycin $\mathrm{C}$ pharmacokinetics during intravesical chemotherapy for the treatment of superficial transitional cell carcinoma. Br J Clin Pharmacol. 2001;52:273-8.

[24] van der Heijden AG, Verhaegh G, Jansen CF, Schalken JA, Witjes JA. Effect of hyperthermia on the cytotoxicity of 4 chemotherapeutic agents currently used for the treatment of transitional cell carcinoma of the bladder: An in vitro study. J Urol. 2005; 173:1375-80.

[25] Lammers RJ, Witjes JA, Inman BA, Leibovitch I, Laufer M, Nativ $\mathrm{O}$, et al. The role of a combined regimen with intravesical chemotherapy and hyperthermia in the management of non-muscle-invasive bladder cancer: A systematic review. Eur Urol. 2011;60:81-93.

[26] Arends TJ, van der Heijden AG, Witjes JA. Combined chemohyperthermia: 10-year single center experience in 160 patients with nonmuscle invasive bladder cancer. J Urol. 2014;192:708-13.

[27] Nativ O, Witjes JA, Hendricksen K, Cohen M, Kedar D, Sidi A, et al. Combined thermo-chemotherapy for recurrent bladder cancer after bacillus Calmette-Guerin. J Urol. 2009;182:1313-7.

[28] Sooriakumaran P, Chiocchia V, Dutton S, Pai A, Ayres BE, Le Roux P, et al. Predictive Factors for Time to Progression after Hyperthermic Mitomycin C Treatment for High-Risk Non-Muscle Invasive Urothelial Carcinoma of the Bladder: An Observational Cohort Study of 97 Patients. Urol Int. 2016;96:83-90

[29] Moskovitz B, Halachmi S, Moskovitz M, Nativ O, Nativ O. 10-year single-center experience of combined intravesical chemohyperthermia for nonmuscle invasive bladder cancer. Future Oncol. 2012;8:1041-9.

[30] Alfred Witjes J, Hendricksen K, Gofrit O, Risi O, Nativ O. Intravesical hyperthermia and mitomycin- $\mathrm{C}$ for carcinoma in situ of the urinary bladder: Experience of the European Synergo working party. World J Urol. 2009;27:319-24.

[31] Sousa A, Pineiro I, Rodriguez S, Aparici V, Monserrat $\mathrm{V}$, Neira $\mathrm{P}$, et al. Recirculant hyperthermic IntraVEsical chemotherapy (HIVEC) in intermediate-high-risk non-muscle-invasive bladder cancer. Int $\mathrm{J}$ Hyperthermia. 2016;32:374-80.

[32] Morales A, Herr H, Steinberg G, Given R, Cohen Z, Amrhein J, et al. Efficacy and safety of MCNA in patients with nonmuscle invasive bladder cancer at high risk for recurrence and progression after failed treatment with bacillus Calmette-Guerin. J Urol. 2015;193:1135-43. 